\title{
Selection of the Position of Temporary Network Routing Node of Wireless Mesh in new Substation
}

\author{
Ping Yu ${ }^{a}$, Yujuan Xue ${ }^{b}$, Jianning Liu ${ }^{c}$
}

North China Electric Power University, Yong hua bei da jie 619, Baoding, Hebei, China

\author{
aemail:yuping63119@yahoo.com.cn \\ bemail:xueyujuan0@163.com \\ cemail:liujianning_9@163.com
}

Keywords: substation of routing node, Okumura-Hata, Mesh, Substation

\begin{abstract}
Use wireless Mesh temporary network to communicate between new substations, in order to quickly build the system network, adopt Okumura-Hata method to compute path loss, and simulate the best location of routing node. Based on the measurement of geographical factors, modify Okumura-Hata model, Matlab made the simulation experiment for prediction model, and obtained path loss in different directions and under different geographical environment. Analyze and draw the system network model from data results, and mark the best location of routing node.
\end{abstract}

\section{Introduction}

On the construction stage, temporary communication system network needs to be established between the new substation and its adjacent substation; the presentation of wireless Mesh communication network makes it more suitable for the characteristics of temporary communication network, and if combined with the requirements of substation communication system, can replace the expensive optical fiber communication.

During the wireless network establishment, topography and other environmental factors have great influence on its transmission, and this makes the propagation path attenuation larger, so not only consider the received signal's strength, but also consider the convenient construction at geographical location, we still need to undertake a lot of work to measure signals, and this extends the network set up time. This paper studies the prediction model of selection of routing node position, predicts its location area, reduces the actual amount of work, and makes it able to quickly build a wireless network.

\section{Prediction of routing node position}

Wireless Mesh ${ }^{[1]}$ network is a wireless local network solution which is very suitable for covering a large open area.

Wireless Mesh network. Wireless Mesh network is also known as the "multi-hop" network, it is a new wireless network technology which is completely different from the traditional wireless networks, and it belongs to the mesh structure.

Mesh network is a dynamic self-organized and self-configured network, and this characteristic has a lot of advantages for end users, and it gradually becomes one of the attention focuses.

Principle for selecting the position of wireless Mesh network routing node. Around an antenna, there should not have obvious obstacles blocking, and the antenna height should be suitable for the area model. Antenna height should be higher than the surrounding buildings. Substation location is at the suburbs, and buildings are not so dense compared with urban buildings, and the routing node position is generally constructed in the hills or slopes, if there are big mountains, avoid them and choose other paths.

The position of routing node may affect the total power of transmission link, in order to reduce the unnecessary power consumption, based on ensuring the communication quality, choose the minimum power zone to establish the routing node ${ }^{[2,3]}$. 
Establishment of predictive model. In the actual engineering, for the specific environment, there is a variety of predictive models for network planning design, but through the simulation comparison between outdoor predictive models, such as Egli, Ibrahim-Parsons, Walfisch-Beroni and Okumura-Hata predictive model ${ }^{[4]}$, the theoretical value measured from Okumura-Hata prediction model is the closest to the actually measured value.

Okumura-Hata model is based on the chart from test data, and it does not provide any explanation, but it should often be modified according to the specific environment parameter ${ }^{[5]}$.

Okumura-Hata model has general characteristics ${ }^{[6]}$, and the path loss formula is:

$$
\mathrm{PL}(\mathrm{dB})=A+B \lg d+C
$$

Tab.1 Okumura-Hata model parameters

\begin{tabular}{|l|l|l|}
\hline Parameter name & Parameter Symbol & Range \\
\hline Carrier frequency & $f_{\mathrm{c}}$ & $150 \sim 1500 \mathrm{MHz}$ \\
The height of the transmitter antenna & $h_{\mathrm{b}}$ & $30 \sim 200 \mathrm{~m}$ \\
The height of the receiver antenna & $h_{\mathrm{m}}$ & $1 \sim 10 \mathrm{~m}$ \\
The distance between the transmitter and receiver & $d$ & $1 \sim 20 \mathrm{~km}$ \\
\hline
\end{tabular}

We have to note that the value range of frequency parameter is given in Table 1 does not fully include $2 \mathrm{G}$ and $3 \mathrm{G}$ band which have higher working frequency ${ }^{[7]}$. In this band, parameter A and B are calculated according to the following definition:

$$
\begin{gathered}
\left\{\begin{array}{l}
\mathrm{A}=46.3+33.9 \lg f_{\mathrm{c}}-13.82 \lg h_{\mathrm{b}}-a\left(h_{\mathrm{m}}\right) \\
\mathrm{B}=44.9-6.55 \lg h_{\mathrm{b}}
\end{array}\right. \\
a\left(h_{\mathrm{m}}\right)=\left(1.1 \lg f_{c}-0.7\right) h_{\mathrm{m}}-\left(1.56 \lg f_{c}-0.8\right) \\
C=-2\left[\lg \left(\frac{f_{\mathrm{c}}}{28}\right)^{2}\right]-5.4
\end{gathered}
$$

In the formula, the unit of carrier frequency $f_{\mathrm{c}}$ is $\mathrm{MHz}$, the unit of receiving and sending $d$ is $\mathrm{km}$, and the unit of height of transmitting antenna $h_{\mathrm{b}}$ and height of receiving antenna $h_{\mathrm{m}}$ is $\mathrm{m}$. The $C$ and $a\left(h_{\mathrm{m}}\right)$ in the formula depend on the working environment of wireless system.

\section{Model simulation and analysis}

We perform practical research for two substations in this study, and their locations are in the suburbs, so adopt the suburb environment as the assessment basis to make survey for the environmental geomorphology. The topography of the source node and destination node diagram shown in Figure 3

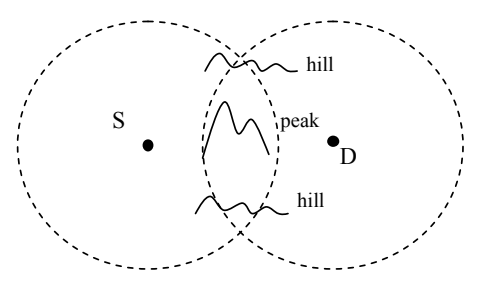

Fig.1 Topographic Map

Figure 1 shows the topographic factor between source node $\mathrm{S}$ and destination node $\mathrm{D}$, and big mountains and hilly areas are the main influence factors for wireless signal.

This article adopts two kinds of Mesh devices, respectively Strix's Access/One outdoor wireless system OWS, and H3C's WH2530X-DAG outdoor system. The parameters shown in Table 2. 
Tab.2 Equipment parameters

\begin{tabular}{|l|l|l|l|}
\hline Model & Transmit power & Receiver sensitivity & Receiver sensitivity \\
\hline Access/One & $26 \mathrm{dBm}$ & $-98 \mathrm{dBm} @ 1 \mathrm{Mbps}$ & $-94 \mathrm{dBm} @ 5.5 \mathrm{Mbps}$ \\
\hline WH2530X-DAG & $20 \mathrm{dBm}$ & $-94 \mathrm{dBm} @ 1 \mathrm{Mbps}$ & $-88 \mathrm{dBm} @ 5.5 \mathrm{Mbps}$ \\
\hline
\end{tabular}

For the system requirement, table 3 shows the system parameters:

Tab.3 System parameters

\begin{tabular}{|l|l|l|}
\hline Parameter name & Parameter Symbol & Value \\
\hline The height of the transmitter antenna & $h_{T X}$ & $50 \mathrm{~m}$ \\
\hline The height of the receiver antenna & $h_{R X}$ & $10 \mathrm{~m}$ \\
\hline Carrier frequency & $f_{\mathrm{c}}$ & $2.4 \mathrm{GHz}$ \\
\hline The distance between the transmitter and receiver & $d$ & $10 \mathrm{~km}$ \\
\hline
\end{tabular}

Influence of big mountain in predictive model. The big isolated peaks have big influence on the signal loss, so we need to add correction factor for the isolated peaks in the Okumura-Hata model. Isolated peaks are equivalent to the edge of obstacles, as shown in Figure 2:

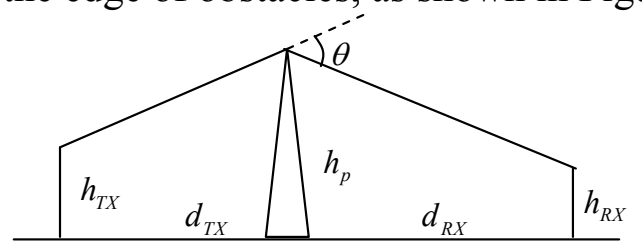

Fig.2 Blade Barrier Geometry Diagram

Calculation of diffraction loss of isolated peaks blade obstacle $K_{p}$ :

$$
K_{p}=6.9+20 \lg \left(\sqrt{(v-0.1)^{2}+1}+v-0.1\right)
$$

Expressions for the parameters:

$$
\begin{gathered}
v=\theta \sqrt{\frac{2 d_{T X} d_{R X}}{\lambda\left(d_{T X}+d_{R X}\right)}} \\
\theta=\arctan \left(\frac{h_{p}-h_{T X}}{d_{T X}}\right)+\arctan \left(\frac{h_{p}-h_{R X}}{d_{R X}}\right)
\end{gathered}
$$

$d_{T X}$ is the distance between source node $\mathrm{S}$ and the peaks, $d_{R X}$ is the distance between destination node and the peaks, they both have $\mathrm{km}$ as unit, and $\lambda$ is the signal wavelength. $h_{p}$ is the vertical height of mountain, $h_{T X}$ and $h_{R X}$ are respectively the antenna height of source node and destination node, with $\mathrm{m}$ as unit.

The predictive formula for direction of source node on big mountain is modified to:

$$
\mathrm{PL}(\mathrm{dB})=A+B \lg d+C+K_{p}
$$

Okumura-Hata model is a formula based on large numbers of experiments and measures, and the predictive data of Okumura-Hata model very meet actually measured data ${ }^{[8]}$. The Measured peak height $h_{p}$ is $232 \mathrm{~m}, d_{T X}$ is $2 \mathrm{~km}, d_{R X}$ is $8 \mathrm{~km}$, and the distance between two substations is bout $10 \mathrm{~km}$. 


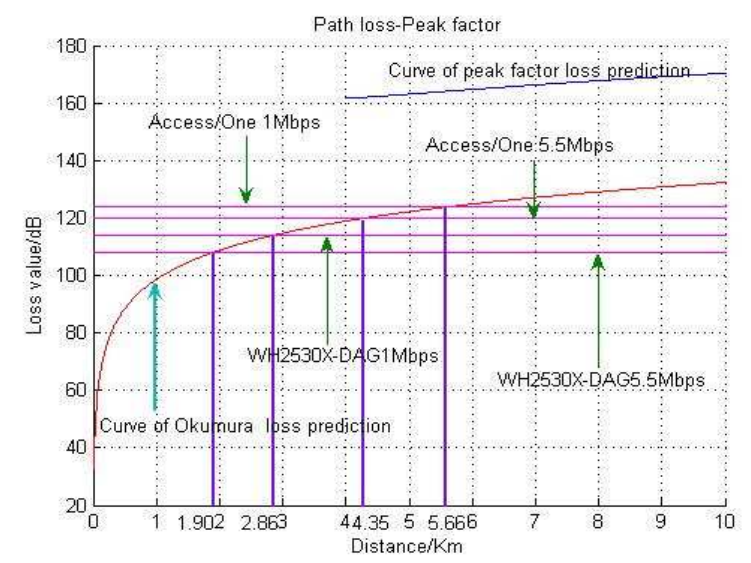

Fig.3 Curve of peak factor loss prediction

In Figure 3, there are two curves, one is not under influence of topographic factors, and the other is added the peak factor. The surface covering length of peaks between source node and destination node is about $2.2 \mathrm{~km}$, and from source node to the foot of mountain, the distance is about $1.8 \mathrm{~km}$. In theory, there should be peak factor at $4 \mathrm{~km}$ from source node behind the mountain, and $1.8 \mathrm{~km} \sim 4 \mathrm{~km}$ is the length of mountain covering surface.

Different devices show different properties in $1 \mathrm{Mbps}$ rate and $5.5 \mathrm{Mbps}$ rate. In the absence of mountain barrier with $1 \mathrm{Mbps}$ rate, Access/One outdoor wireless system's maximum transmission distance is $5.66 \mathrm{~km}$, and WH2530X-DAG outdoor system's distance is $2.86 \mathrm{~km}$. While in $5.5 \mathrm{Mbps}$ rate, Access/One outdoor wireless system's transmission distance will change to $4.35 \mathrm{~km}$, and WH2530X-DAG outdoor system's distance is $1.902 \mathrm{~km}$. While the distance between source node and destination node is $10 \mathrm{~km}$. From the data obtained, we can use single strand to build network, Access/One outdoor building system in $1 \mathrm{Mbps}$ rate requires at least one routing node, while in $5.5 \mathrm{Mbps}$ rate, it requires two. WH2530X-DAG outdoor system in $1 \mathrm{Mbps}$ rate requires at least four routing nodes, while in $5.5 \mathrm{Mbps}$ rate; it requires at least five routing nodes.

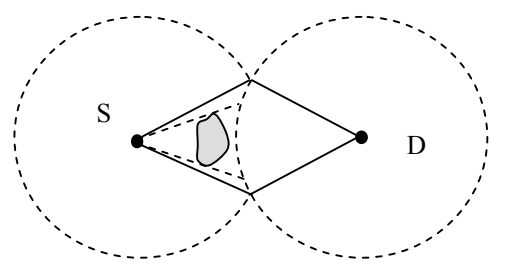

Fig.4 Network diagram of Peak effect

Figure 4 shows that the grey parts are blocked by mountains, and two dotted lines indicate that in the mountain barrier direction, the effective transmission distance of the used equipment for study can't reach the locations behind the mountain, and the covering radius of two nodes can't intersect in this direction, therefore, in this direction it is not good to select the location for the routing node.

Influence of hilly terrain in predictive model

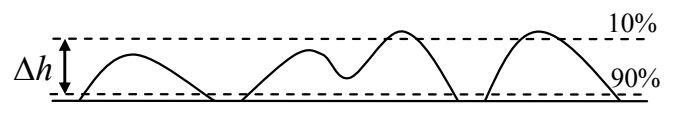

Fig.5 Hilly terrain map

In Figure 5, $\Delta h$ is the difference between $10 \%$ and $90 \%$ of the undulating terrain, and it requires the undulating times greater than 3 .

Then the $K_{h}$ that Calculation of the hilly terrain loss is:

$$
K_{h}=\left\{\begin{array}{cc}
0 & \Delta h<15 \\
-(-5.7+0.024 \Delta h+6.96 \lg \Delta h)-\left(9.5 \lg h_{1}-7.2\right) & \Delta h \geq 15, h_{1}>1 \\
-(-5.7+0.024 \Delta h+6.96 \lg \Delta h)+7.2) & \Delta h \geq 15, h_{1} \leq 1
\end{array}\right.
$$


In formula (13), $h_{1}=h_{m g}-\Delta h / 8-h_{\min }, h_{m g}$ and $h_{\min }$ are respectively the highest and lowest terrain height on calculation profile $\Delta h$. The onsite measurements are $\Delta h$ as $20 \mathrm{~m}$ and $h_{\min }$ as $10 \mathrm{~m}$.

The direction prediction formula of source node in a hilly is modified to:

$$
\mathrm{PL}(\mathrm{dB})=A+B \lg d+C+K_{h}
$$

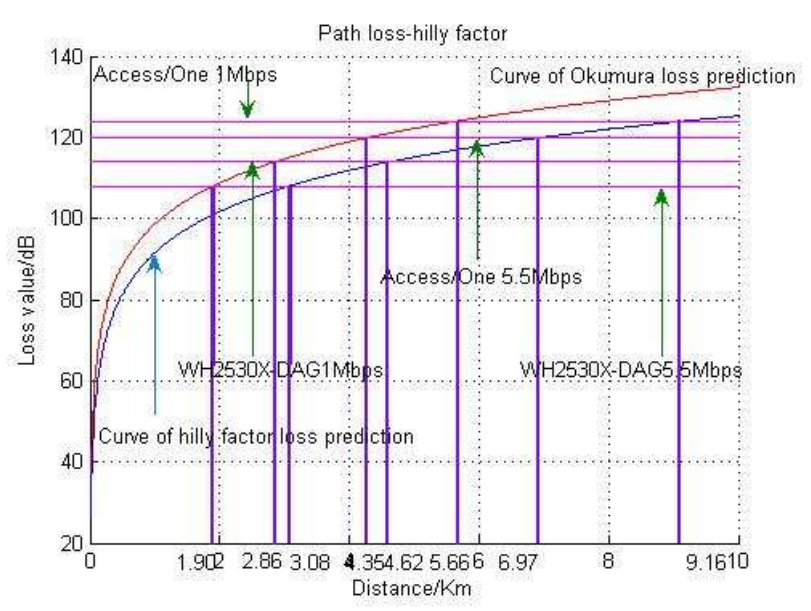

Fig.6 Curve of hilly factor loss prediction

Similar to figure 3, figure 6 also has two prediction curves, one is not under influence of topographic factors, and the other is added prediction curve of hilly factor. In the absence of the influence of terrain, the curve is consistent with the one in figure 3.

After adding the hilly factor, in the allowed loss of device, the effective propagation distance changes. Access/One outdoor wireless system in $1 \mathrm{Mbps}$ rate has the maximum transmission distance as $9.16 \mathrm{~km}$, and WH2530X-DAG outdoor system has the distance as $4.63 \mathrm{~km}$. When the rate changes to $5.5 \mathrm{Mbps}$, Access/One outdoor wireless system has the distance as $6.97 \mathrm{~km}$, and WH2530X-DAG outdoor system has the distance as $3.08 \mathrm{~km}$. From the data obtained, we can use single strand to build network, Access/One outdoor building system in both rates requires at least one routing node, while in $1 \mathrm{Mbps}$ rate, WH2530X-DAG outdoor system requires at least two routing nodes, while in $5.5 \mathrm{Mbps}$ rate it requires at least three routing nodes.

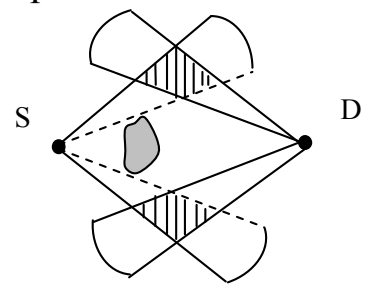

Fig.7 Network diagram of hill effect

in figure 7,we consider the influence of hill factors, two nodes intersect in hilly direction within Access/One outdoor system's effective communication distance, and the shadow part is the best location of routing node. While WH2530X-DAG outdoor system doesn't have intersection in hill direction within communication distance, this shows one routing node can't meet requirements of communication system.

\section{Conclusions}

This article uses Okumura-Hata model to research different geographic environment and make correction, Matlab simulation predicts the propagation path loss of wireless signal under different circumstances in this project, and compare with the different equipment communication performance, draw the covering area of the transmitting and receiving antenna, and obtain the location area of routing node. The method in this article can further accurate position of routing node, accelerate construction progress, save construction cost, and provide a theoretical method for the selection of position of routing nodes. 


\section{References}

[1] Mahesh K M, Samir R D, and Anand P S.A topology control approach for utilizing multiple channels in multi- radio wireless mesh networks [J]. Computer Networks, 2010,54(2):241-256

[2] Host-Madsen A, Zhang J. Capacity bounds and power allocation for wireless relay channels[J]. IEEE Trans. on Information Theory, 2005, 51(6): 2020-2040.

[3] Hasna M O, Alouini M. Optimal power allocation for relayed transmissions over Rayleigh fading channels[J]. IEEE Trans. On Wireless Communications, 2004, 3(6): 1999-2004.

[4] Hata, Masahuaru, Empirical Formula for Propation Loss in Land Mobile Radio Services[J]. IEEE Transactions on Vehicular Technology, August 1980,29(03): 317-325.

[5] ZHANG Yan- hua, DUAN Zhan-yun, SHEN Lan-sun, SHEN Jian, ZHANG Jian. Okumura-Hata radio propagation models using visual simulation[J]. CHINESE JOURNAL OF RADIO SCIENCE, 2001,16(1):89-92

[6] Wei Chong-yu. Basis and Application of Wireless Communication[M]. Xian : XiDian University Press, 2009.8

[7] European Cooperation in the Field of Scientific and Technical Research EURO-COST 231. Urban Transmission Loss Models for Mobile Radio in the 900 and $1800 \mathrm{MHz}$ Bands [ R ]. Revision 2, The Hague, September 1991.

[8] ZHANG Xin, YANG Ming-hua. Adjustment and Realization of Okumura-Hata Prediction Mode in Suburb Environment[J]. Communications Technology, 2008,5(41):66-68 